\title{
VALIDAÇÃO DO MÉTODO DE DETERMINAÇÃO SIMULTÂNEA DOS ÍONS BROMETO E BROMATO POR CROMATOGRAFIA IÔNICA EM ÁGUAS DE CONSUMO HUMANO*
}

\author{
Maria Edite Pinto Gonçalves* e Maria Filomena Camões
}

Faculdade de Ciências, Universidade de Lisboa - Centro de Electroquímica e Cinética da Universidade de Lisboa /Departamento de Química e Bioquímica, Edifício C8, $3^{\circ}$ piso, Campo Grande 1749-016 Lisboa - Portugal

João Miguel Paiva, Maria João Benoliel e Rui Neves Carneiro

Empresa Portuguesa das Águas Livres, Rua do Alviela,12 Calçada dos Barbadinhos, 1170-012 Lisboa - Portugal

Recebido em 19/5/03; aceito em 5/8/03

\begin{abstract}
VALIDATION OF SIMULTANEOUS DETERMINATION OF BROMIDES AND BROMATES BY ION CHROMATOGRAPHY IN DRINKING WATER. European Directive (98/83/CE), compulsory after 2008, states that bromate in drinking water must be controlled at levels below $10 \mathrm{mg} \mathrm{L}^{-1}$. Supporting implementation of the Directive, the European Comission has established project SMT4-CT96 2134, in collaboration with various european institutions, aiming at the identification of the interferents to the current analytical method (Ionic Chromatography with Conductimetric Detection - IC/CD), their removal and the automation of pretreatment and injection steps, as well as the development of alternative methods. EPAL, responsible for the water supply to a great deal of Portuguese regions, has taken steps to meet these requirements. Although not part of such project, this work (the result of a project conducted under a protocol of collaboration between EPAL SA and FCUL - Faculty of Sciences, University of Lisbon), reports on studies of usefulness to laboratories planning to monitor bromate in ozone treated waters, in conditions different from those described in EPA 300.1. Simultaneous determination of bromide is justified by its role as bromate precursor.
\end{abstract}

Keywords: ion chromatography; ozone treatment; water analysis, bromide and bromate.

\section{INTRODUÇÃO}

A etapa de desinfecção no processo de tratamento de água é, sem dúvida, a mais importante quando se trata de água destinada ao consumo humano. Ao longo de muitos anos, o agente de desinfecção utilizado foi o cloro, mais especificamente na forma de ácido hipocloroso $(\mathrm{HOCl})$, substância altamente tóxica, cujos efeitos residuais, e a formação de subprodutos tóxicos, alguns dos quais cancerígenos, têm enfatizado a necessidade de se considerar métodos alternativos de desinfecção, como é o caso da ozonização.

Na ozonização, o agente desinfetante é o gás ozônio $\left(\mathrm{O}_{3}\right)$ que, por sua vez, também origina subprodutos tóxicos, de entre os quais os íons bromato $\left(\mathrm{BrO}_{3}^{-}\right)$que têm origem na oxidação dos íons brometo $\left(\mathrm{Br} \mathrm{r}^{-}\right)$presentes de forma natural nas águas a serem tratadas.

Existem vários mecanismos documentados na literatura, que procuram demonstrar a forma com que os íons brometo são oxidados a íons bromato pela ação do ozônio.

Segundo von Gunten e Hoigné1, em estudos mecanísticos realizados em amostras de águas simuladas, "não naturais", demonstraram que na presença de um captador orgânico para os radicais $\mathrm{OH}$, a formação do bromato pode ser predita com exatidão através de uma oxidação direta, mesmo em águas contendo amônia:

$\mathrm{Br}^{-}+\mathrm{O}_{3}+\mathrm{H}_{2} \mathrm{O} \rightarrow \mathrm{HOBr}+\mathrm{O}_{2}+\mathrm{OH}$

$\mathrm{HOBr}+\mathrm{H}_{2} \mathrm{O} \rightarrow \mathrm{H}_{3} \mathrm{O}^{+}+\mathrm{OBr}$

$\mathrm{OBr}+\mathrm{O}_{3} \rightarrow \mathrm{Br}^{-}+2 \mathrm{O}_{2}$

$\mathrm{OBr}^{-}+2 \mathrm{O}_{3} \rightarrow \mathrm{BrO}_{3}^{-}+2 \mathrm{O}_{2}$

$\mathrm{HOBr}+\mathrm{O}_{3} \rightarrow$ reação muito lenta !

*e-mail: megoncalves@fc.ul.pt

\#As opiniões expressas são da inteira e exclusiva responsabilidade dos autores, não configurando a posição oficial da EPAL, S.A. sobre a matéria.
$\mathrm{Na}$ ausência destes captadores, as reações radicalares do $\mathrm{OH}$ contribuem significativamente na formação dos bromatos por oxidação indireta.

$\mathrm{O}$ íon bromato pode, portanto, ser formado tanto pela ação direta do ozônio molecular, quanto pela ação do radical hidroxilo sobre o íon brometo.

Estudos mais recentes por Westerhoff et al. ${ }^{2}$ realizados em amostras de águas naturais (contendo matéria orgânica) e não naturais (livres de matéria orgânica), concluíram que a formação de bromato pelos mecanismos do ozônio molecular e do radical $\mathrm{OH}$ pode variar de uma amostra para outra dependendo do $\mathrm{pH}$ e do carbono orgânico dissolvido. Em amostras de água naturais contendo matéria orgânica, a formação de bromato é controlada pelo mecanismo do radical $\mathrm{OH}$, enquanto em amostras de águas simuladas (não naturais) livres de carbono orgânico dissolvido, ambos os mecanismos são importantes numa proporção de $30 \%$ para o mecanismo do ozônio molecular e $70 \%$ para o do radical $\mathrm{OH}$.

Provenientes da oxidação dos íons brometo $\left(\mathrm{Br}^{-}\right)$presentes nas águas a serem tratadas, os íons bromato $\left(\mathrm{BrO}_{3}^{-}\right)$são os que mais preocupações trazem à Organização Mundial de Saúde $(\mathrm{OMS})^{3}$ bem como a outras agências reguladoras como a U.S. EPA (Agência de Proteção Ambiental dos Estados Unidos) ${ }^{4}$ devido ao seu carácter cancerígeno, mesmo a níveis de poucos $\mu \mathrm{g} \mathrm{L}^{-1}$. Dentro da União Européia (UE), a Diretiva Comunitária da Água ${ }^{5}$, (98/83/CE) de 3 de Novembro de 1998 (relativa à qualidade da água destinada ao consumo humano), reflete essa preocupação recomendando, de acordo também com a OMS e a U.S. EPA, que os níveis de íons bromato presentes estejam abaixo dos $10 \mu \mathrm{g} \mathrm{L}^{-1}$. Os Estados membros têm um prazo de dez anos para se adequarem a este valor estabelecido. Entretanto, no período compreendido entre os cinco e os dez anos após a entrada em vigor da diretiva, um valor paramétrico provisório de $25 \mu \mathrm{g} \mathrm{L}^{-1}$ vigorará, enquanto estudos de risco de saúde mais aprofundados vão sendo realizados.

Diante deste quadro, a necessidade de implementação, por parte 
dos órgãos nacionais responsáveis pela monitorização da qualidade da água nos países membros da UE, de um método analítico capaz de detectar e quantificar os íons bromato nestes níveis de concentração, é de extrema importância, o que levou a EPAL (Empresa Portuguesa das Águas Livres), entidade responsável pela qualidade da água consumida no território português, a implementar e validar um método analítico capaz de detectar e quantificar os íons bromato a estes níveis de concentração, e assim cumprir o estabelecido na Directiva Comunitária.

Neste trabalho aqui descrito, foi estudada a determinação simultânea dos íons brometo e bromato através de Cromatografia Iônica. Esta metodologia escolhida, ainda em fase de desenvolvimento técnico e científico, após pesquisa na literatura de métodos analíticos existentes adequados, foi submetida a um extenso estudo de validação, através de testes alusivos ao intervalo de trabalho, limites de detecção e quantificação, linearidade, sensibilidade e ensaios de recuperação.

Com algumas adaptações às condições existentes, o método foi aplicado, e os resultados alcançados permitem à EPAL cumprir as exigências colocadas pela Diretiva Comunitária.

O interesse na determinação simultânea dos íons brometo é devido ao seu papel de precurssor na formação dos íons bromato como demonstrado anteriormente.

\section{PARTE EXPERIMENTAL}

\section{Sistema cromatográfico}

As análises cromatográficas foram realizadas num cromatógrafo modelo DX-120 (Dionex Sunnyvale, CA) equipado com bomba isocrática. $\mathrm{O}$ volume de injeção da amostra era de $500 \mu \mathrm{L}$, as colunas analíticas incluíam uma pré-coluna Dionex AG9-HC (50x4 mm), e uma coluna analítica Dionex AS9-HC (250x4 mm). Detecção obtida por um detector de condutividade - célula CDM-3 Dionex com volume interno de 1,0 $\mu \mathrm{L}$. A condutividade do eluente foi suprimida através do supressor aniônico Dionex ASRS II (4 mm) no modo de regeneração por auto-supressão, aplicando-se uma corrente ao supressor de $300 \mathrm{~mA}$. A aquisição e tratamento de dados foi obtida através do software PeakNet 5.01 (Dionex).

\section{Reagentes e soluções}

Todas as soluções aquosas foram preparadas com água deionizada, ultrapura tipo I, filtrada por membrana $0,22 \mu \mathrm{m}$ obtida com um sistema Milli-Q (Millipore, Bedford, MA, USA). O eluente $\mathrm{Na}_{2} \mathrm{CO}_{3}$ 9,0 $\mathrm{mM}$ (Merck) foi purgado em ultrassons durante $15 \mathrm{~min}$ para remoção dos gases dissolvidos (principalmente $\mathrm{CO}_{2}$ ). As soluções padrão dos analitos, $\mathrm{NaBr}$ (Fluka Chemika) e $\mathrm{NaBrO}_{3}$ (SigmaAldrich-Chemie), foram preparadas através da diluição das soluções padrão-mãe de $1.000 \mathrm{mg} \mathrm{L}^{-1}$ (ppm).

\section{RESULTADOS E DISCUSSÃO}

Inicialmente o método escolhido (U. S. EPA - método 300.1 Determinação de ânions inorgânicos em água para consumo humano por cromatografia iônica) ${ }^{6}$ e adaptado às condições existentes, foi testado com relação aos seus limites de detecção.

Os primeiros testes para a verificação da resposta analítica do cromatógrafo e da coluna analítica foram realizados com a solução 9,0 mM de $\mathrm{Na}_{2} \mathrm{CO}_{3}$ como eluente com uma vazão de $1,25 \mathrm{~mL} \mathrm{~min}^{-1}$ e um volume de injeção de amostra de $200 \mu \mathrm{L}$.

Os resultados iniciais demonstraram que a sensibilidade do aparelho não atingia os níveis de concentração desejados, principalmente com relação aos bromatos, cuja detecção era facilmente confundida com o ruído da linha de base, além de ser também prejudicada pela interferência dos íons cloreto, cujo tempo de retenção é bastante próximo ao dos íons bromato.

Através da alteração do volume de amostra injetado de $200 \mu \mathrm{L}$ para $500 \mu \mathrm{L}$, foi obtida uma melhora significativa na sensibilidade do aparelho, o que se refletiu no limite de detecção alcançado.

A partir destes testes iniciais pode-se definir e estudar uma gama de trabalho, e proceder-se a construção das curvas de calibração para os íons brometo e bromato em simultâneo.

\section{Intervalo de trabalho}

Ao final de inúmeras tentativas com vários intervalos, cujos testes de homogeneidade de variâncias se mostraram insatisfatórios, chegou-se ao estabelecimento dos intervalos, 50 a $500 \mu \mathrm{g} \mathrm{L}^{-1}$ e 10 a $50 \mu \mathrm{g} \mathrm{L}^{-1}$ para brometo e bromato, respectivamente, utilizando um volume de amostra de $200 \mu \mathrm{L}$ como recomenda a literatura ${ }^{6}$. Após alguns testes decidiu-se alterar o volume de amostra para $500 \mu \mathrm{L}$. Vale ressaltar também que, a fim de obtermos melhores resultados na determinação conjunta destes íons, a faixa de trabalho que satisfez os testes estatísticos em simultâneo, foi aquela que se apresenta acima. A faixa de trabalho estabelecida para os brometos também satisfaz a zona de concentrações deste íon que deve ser monitorizada, como demonstra um estudo ${ }^{7}$ que revelou durante experimentos de ozonização em águas tratadas por coagulação e filtração, que concentrações de brometo tão baixas quanto os $50 \mu \mathrm{g} \mathrm{L}^{-1}$ são suficientes para promover a formação de bromato. $\mathrm{O}$ mesmo estudo refere que concentrações de brometo entre os 100 e os $500 \mu \mathrm{g} \mathrm{L}^{-1}$ produzem íons bromato em águas ozonizadas acima dos limites estabelecidos pela Diretiva Européia.

Os intervalos de trabalho estabelecidos foram estudados em simultâneo através da utilização de cinco pontos de calibração distribuídos regularmente ao longo dos respectivos intervalos. Para o estudo da homogeneidade de variâncias foram construídas curvas de calibração através da injeção de padrões mistos, num volume de $500 \mu \mathrm{L}$, obtendo-se cromatogramas semelhantes aos que constam da Figura 1, onde os picos relativos aos bromatos são aqueles cujo tempo de retenção é de aproximadamente 4,9 min, e os picos relativos aos brometos são aqueles cujo tempo de retenção é de aproximadamente 8,6 min. Os picos cujo tempo de retenção é de aproximadamente 5,4 min devem-se aos íons cloreto, um interferente importantíssimo nas determinações de bromato, uma vez que ambos os íons eluem da coluna analítica em tempos muito próximos.

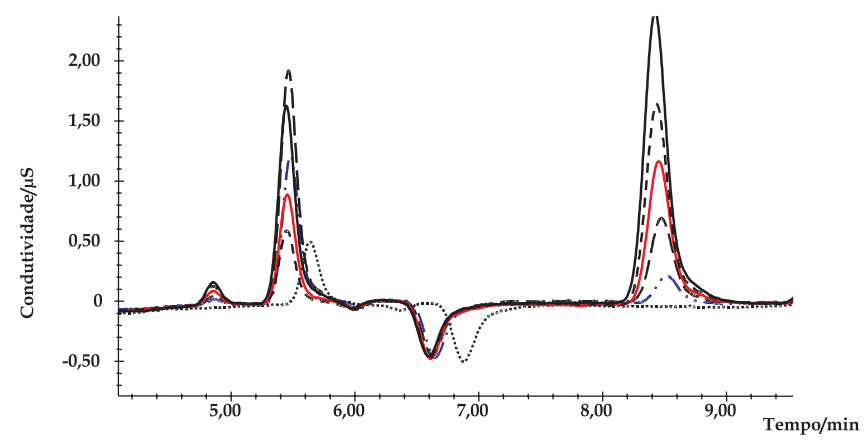

Figura 1. Cromatogramas obtidos para os ions Brometo ( $\left.\boldsymbol{t}_{r} 8,6 \mathrm{~min}\right) \mathrm{na}$ gama de 0,05 a 0,50 $\mathrm{mg} \mathrm{L}^{-1}$, e Bromato ( $\boldsymbol{t}_{r}$ 4,9 min) na gama de 0,01 a

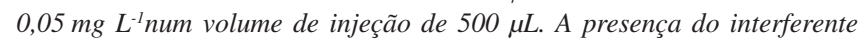
cloreto nota-se no $\boldsymbol{t}_{r} 5,9 \mathrm{~min}$

As curvas de calibração obtidas para os íons brometo e bromato, a partir das áreas sob os picos relativos às concentrações dos padrões, apresentam-se respectivamente nas Figuras 2 e 3. 


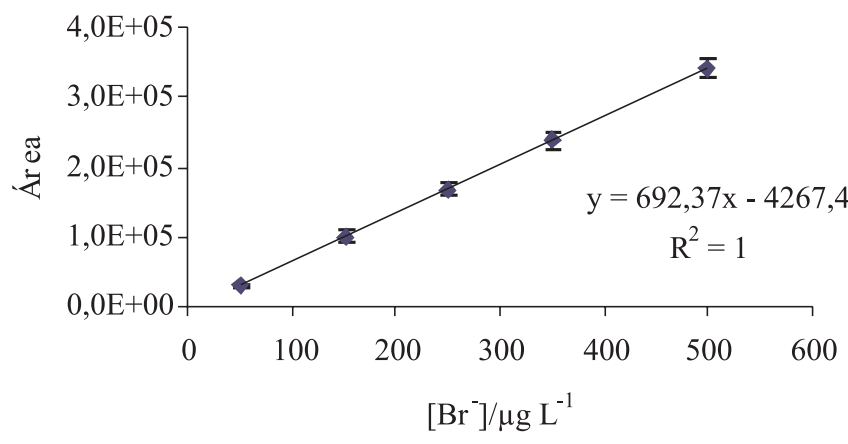

Figura 2. Gráfico da função de calibração dos brometos na gama de 0,05 a $0,50 \mathrm{mg} \mathrm{L}^{-1}$; estudo da linearidade

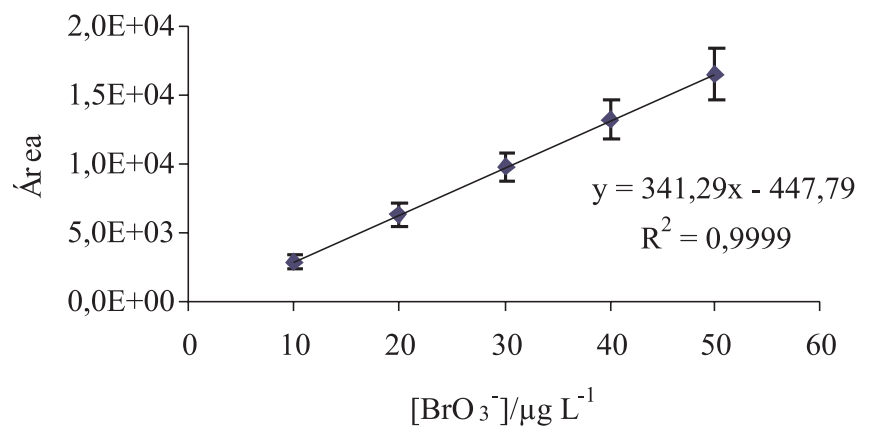

Figura 3. Gráfico da função de calibração dos bromatos na gama de 10 a

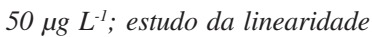

A homogeneidade de variâncias dos intervalos de trabalho foi testada de maneira independente durante três dias, através do Teste de Fisher $^{8}$ (onde o primeiro e o último padrão das curvas de calibração para ambos os íons foram analisados em 10 réplicas independentes e

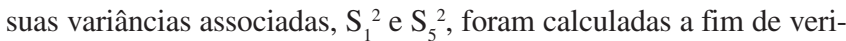
ficar a existência ou não de diferenças significativas entre elas). $\mathrm{O}$ valor teste $\mathrm{PG}$ obtido através da razão destas variâncias é comparado com o valor tabelado da distribuição F de Snedecor/Fisher (neste caso $\mathrm{F}(9 ; 9 ; 0,99)=5,35)$.

A linearidade foi testada através do Teste de Mandel $^{8,9}$ (onde estimamos qual dos modelos - linear ou não-linear - proporciona o melhor ajuste dos pontos da curva de calibração). $\mathrm{O}$ valor teste $\mathrm{PG}$, é comparado com o valor tabelado da distribuição F de Snedecor/ Fisher (neste caso $\mathrm{F}(4 ; 4 ; 0,99)=15,98$ ).

Na Tabela 1, apresentam-se os resultados em termos numéricos dos testes de linearidade e homogeneidade de variâncias.

De acordo com estes resultados, os valores obtidos para os testes de Fisher mostram que não há diferenças significativas entre as variâncias dos padrões-limites, ou seja, as variâncias são homogêneas e independentes da concentração ao longo de todo o intervalo
Tabela 1. Resultado dos estudos de linearidade e homogeneidade

\begin{tabular}{lccc}
\hline Parâmetros & Critério & $\begin{array}{c}\text { Brometo } \\
\text { média }\end{array}$ & $\begin{array}{c}\text { Bromato } \\
\text { média }\end{array}$ \\
\hline Homogeneidade de variâncias & $\mathrm{PG}<5,35$ & 2,59 & 3,8 \\
Linearidade & $\mathrm{PG}<15,98$ & 3,26 & 1,27 \\
Coeficiente de correlação $(\mathrm{R})$ & $(\mathrm{R})>0,995$ & 1,000 & 0,9999 \\
\hline
\end{tabular}

estabelecido para cada um dos íons, os resultados demonstram também que o modelo linear é o que melhor se ajusta aos pontos obtidos, o que também se confirma através dos coeficientes de correlação (R) obtidos, muito próximos de 1,000.

\section{Limiares Analíticos - Limites de Detecção (LDM) e de Quantificação (LQM)}

Os limiares analíticos foram calculados estatisticamente ${ }^{8,10} \mathrm{de}$ acordo com as equações (1) e (2), e os resultados obtidos encontram-se na Tabela 2.

$\operatorname{LDM}=\frac{\left[3,3 \cdot S_{y / x}\right]}{b}$

$\mathrm{LQM}=\frac{\left[10 \cdot S_{y / x}\right]}{b}$

onde $S_{y / x}$ é o desvio padrão residual da curva de calibração e b é o declive da mesma.

Analisando os resultados que constam da Tabela 2 com relação ao brometo, nota-se que, de maneira geral, os limites de quantificação encontram-se ligeiramente acima da concentração do primeiro padrão, mas estes resultados são muito melhores do que os apresentados em intervalos estabelecidos em tentativas anteriores. O limite de detecção médio obtido de $16,4 \mu \mathrm{g} \mathrm{L} \mathrm{L}^{-1}$, está muito acima dos $0,98 \mu \mathrm{g}$ $\mathrm{L}^{-1}$ registrados na literatura para a metodologia escolhida ${ }^{6}$.

O bromato, que é na realidade o objeto de interesse deste estudo, apresenta de modo geral, resultados muito melhores em relação aos limites de detecção e quantificação do que os apresentados pelo brometo. De acordo com a Tabela 2, nota-se que o limite de quantificação médio obtido para o bromato de $6,7 \mu \mathrm{g} \mathrm{L}^{-1}$, encontrase abaixo da concentração do primeiro padrão que é de $10 \mu \mathrm{L} \mathrm{L}^{-1}$, portanto abaixo do valor paramétrico limite estabelecido na Diretiva. Com relação ao limite de detecção médio obtido de $2,2 \mu \mathrm{g} \mathrm{L} \mathrm{L}^{-1}$, é extremamente satisfatório considerando que o valor registrado na literatura é de $1,32 \mu \mathrm{g} \mathrm{L}{ }^{-1}$. Sendo o objetivo principal deste trabalho alcançar um nível de detecção dos íons bromato em concentrações bastante baixas a fim de se poder detectar a sua presença nos níveis considerados atualmente prejudiciais à saúde, os resultados alcançados são bastante satisfatórios no que diz respeito aos limiares analíticos.

Tabela 2. Limiares analíticos do método para os íons brometo e bromato

\begin{tabular}{|c|c|c|c|}
\hline Parâmetros & Critério & $\begin{array}{c}\text { Brometo } \\
\text { média }\end{array}$ & $\begin{array}{c}\text { Bromato } \\
\text { média }\end{array}$ \\
\hline $\mathrm{LDM} / \mu \mathrm{g} \mathrm{L} \mathrm{L}^{-1}$ & - & 16,4 & 2,2 \\
\hline $\mathrm{LQM} / \mu \mathrm{g} \mathrm{L} \mathrm{L}^{-1}$ & $\begin{array}{c}\mathrm{LQM}<50 \mu \mathrm{g} \mathrm{L}^{-1} / \mathrm{Br}^{-} \\
\mathrm{LQM}<10 \mu \mathrm{g} \mathrm{L}^{-1} / \mathrm{BrO}_{3}^{-}\end{array}$ & 49,0 & 6,7 \\
\hline Sensibilidade (b) & - & 692371,6 & 341288,7 \\
\hline Desvio Padrão Residual $\left(\mathrm{S}_{\mathrm{y} / \mathrm{x}}\right)$ & - & 3431,47 & 223,86 \\
\hline Coef. de Variação do método $\left(\mathrm{V}_{\mathrm{x} 0}\right) \%$ & $\left(\mathrm{~V}_{\mathrm{x} 0}\right)<5 \%$ & 1,91 & 2,25 \\
\hline
\end{tabular}


O aumento no volume de amostra elevou a amplitude do sinal analítico obtido (o que se denota através da inclinação da reta obtida) trazendo uma grande melhora na sensibilidade do método, tendo como conseqüência o abaixamento do limite de detecção e de quantificação do método, tornando possível detectar a presença do íon bromato nos níveis de concentração abaixo dos exigidos pela legislação que entrará em vigor.

Apesar dos valores de sensibilidade (b) obtidos para o brometo serem muito mais elevados do que os obtidos para os bromatos, os valores do desvio padrão residual (que descreve a precisão da calibração) apresentados pelos brometos também são muito mais elevados do que os do bromato, denotando um grande desvio dos valores experimentais com relação aos valores estimados pela reta de regressão. Traduzindo-se, no caso dos brometos, em piores limites de detecção e quantificação com relação aos alcançados pelos bromatos.

Os resultados obtidos para o coeficiente de variação do método $\left(V_{x 0}\right) \%$ (Equação 3), que se encontram na Tabela 2, são bastante satisfatórios permitindo ao analista verificar o seu desempenho.

$V_{x 0}=\frac{S_{y / x}}{b} \cdot \frac{1}{\bar{x}} \cdot 100$

Estudos de exatidão com materiais de referência certificados, não puderam ser realizados pelo fato de, na ocasião, não existirem estes materiais disponíveis para os íons brometo e bromato.

Os ensaios de recuperação para o bromato foram realizados em algumas amostras em que a etapa de desinfecção foi realizada através da ozonização. As amostras foram coletadas em uma estação de tratamento de água nas etapas anterior e posterior ao processo de ozonização, e após uma primeira análise para determinação da concentração inicial do íon bromato, estas foram fortificadas através da adição à amostra de uma quantidade conhecida do analito (ver Tabela 3). Os resultados obtidos para estes ensaios de recuperação encontram-se na Tabela 3.

De acordo com o Standard Methods ${ }^{11}$, a porcentagem de recuperação pode variar de 80 a $120 \%$. Qualquer valor obtido compreendido dentro deste intervalo fornece a informação de que tanto o procedimento quanto a matriz não influenciam de modo significativo na detecção do analito, demonstrando que o método é capaz de identificar todo o analito presente.

Apesar das poucas amostras analisadas, os valores obtidos nos ensaios de recuperação encontram-se na sua maioria dentro da faixa dos 80 aos $120 \%$. Os resultados demonstram que o procedimento escolhido não afeta a detecção dos bromatos.

No caso da amostra pré-ozonizada, o valor de recuperação obtido, $75,5 \%$, poderá estar associado a características fracamente oxidantes do meio com reflexo na menor estabilidade do ion bromato.

\section{Efeito do uso da etilenodiamina na determinação dos íons bromato}

A metodologia utilizada, recomenda a utilização da etileno- diamina (EDA), para preservação das amostras no caso da determinação de bromato ${ }^{6}$.

A adição de EDA na concentração de $50 \mathrm{mg} \mathrm{L}^{-1}$ nas amostras tem como objetivo impedir que íons hipobromito $\left(\mathrm{OBr}^{-}\right)$, possivelmente presentes em águas ozonizadas, uma vez que são intermediários nas reações de oxidação dos íons brometo a bromato, venham com o passar do tempo a transformar-se em bromato, alterando a sua concentração original na amostra. Sendo assim, os padrões de calibração, devem estar nas mesmas condições das amostras. Portanto, a EDA deve ser adicionada aos padrões na concentração de $50 \mathrm{mg} \mathrm{L}^{-1}$.

Devido às dúvidas com relação a adição de EDA vir a deteriorar a coluna analítica, e também interferir na determinação dos íons bromato, foram realizados testes que consistiram na preparação dos padrões de calibração, adicionando etilenodiamina na concentração de $50 \mathrm{mg} \mathrm{L}^{-1}$.

As injeções dos padrões foram realizadas normalmente, notando-se através dos cromatogramas obtidos (de acordo com a Figura 4) que, de uma injeção para outra, um pico nunca detectado até então, surgiu próximo ao tempo de retenção dos cloretos e que a cada injeção se tornava maior, independente do fato de a concentração adicionada de EDA ser sempre a mesma.

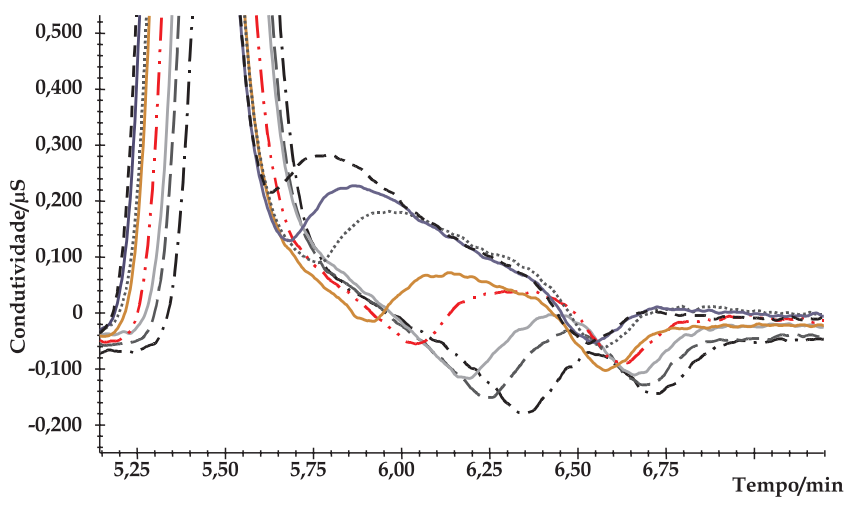

Figura 4. Efeito cumulativo da Etilenodiamina na coluna analítica

Conclui-se, que a etilenodiamina vai se acumulando na coluna analítica diminuindo, com o passar do tempo, os sítios ativos disponíveis na coluna, afetando deste modo as posteriores determinações quantitativas dos analitos de interesse.

Decidiu-se pela não utilização de etilenodiamina como conservante das amostras. Aconselhando-se então que as amostras sejam analisadas no período de uma semana, mantendo-as sob refrigeração a $4{ }^{\circ} \mathrm{C}$.

\section{CONCLUSÃO}

De acordo com os resultados obtidos nos estudos de validação do método de determinação simultânea dos íons brometo e bromato, nos intervalos de 50 a $500 \mu \mathrm{g} \mathrm{L}^{-1}$ e de 10 a $50 \mu \mathrm{g} \mathrm{L}^{-1}$, respectivamen-

Tabela 3. Ensaio de recuperação - íons bromato

\begin{tabular}{lcccccc}
\hline Amostra & $\begin{array}{c}\text { Vol. } \\
\text { amostra } \\
(\mathrm{mL})\end{array}$ & $\begin{array}{c}\text { Vol. } \\
\text { padrão } \\
(\mathrm{mL})\end{array}$ & $\begin{array}{c}\text { Conc. inicial } \\
\text { amostra } \\
(\mu \mathrm{g} / \mathrm{L})\end{array}$ & $\begin{array}{c}\text { Conc. } \\
\text { do padrão } \\
(\mu \mathrm{g} / \mathrm{L})\end{array}$ & $\begin{array}{c}\text { Conc. obtida } \\
\text { amostra+padrão } \\
(\mu \mathrm{g} / \mathrm{L})\end{array}$ & $\begin{array}{c}\text { Recuperação } \\
\text { do padrão } \\
(\%)\end{array}$ \\
\hline Pré-ozonização 17/08 & 24,875 & 0,125 & 15,0 & 50,0 & 49,0 & 75,5 \\
Pós-ozonização 17/08 & 24,875 & 0,125 & 14,0 & 50,0 & 57,6 & 90,0 \\
Desinfecção final 17/08 & 24,875 & 0,125 & 14,0 & 50,0 & 58,8 & 92,0 \\
\hline
\end{tabular}


te, pode-se dizer que o objetivo inicialmente proposto de implementação de um método analítico por Cromatografia Iônica, para determinação quantitativa de brometo e bromato na faixa de concentração necessária às exigências da Diretiva Comunitária da Água 98/83/CE de 3 de Novembro de 1998, foi alcançado.

O método implementado, satisfez as exigências com relação ao bromato, com um limite de detecção médio de $2,2 \mu \mathrm{g} \mathrm{L} \mathrm{L}^{-1}$, e um limite de quantificação médio de $6,7 \mu \mathrm{g} \mathrm{L}^{-1}$; abaixo do valor máximo recomendado de $10 \mu \mathrm{g} \mathrm{L}^{-1}$. De acordo com estes resultados, conclui-se também que a presença do íon cloreto não causou interferência real à análise do íon bromato.

Com relação ao brometo, não há um valor de concentração a ser respeitado, mas sua determinação é importante, devido ao seu papel de precursor na formação do bromato.

O método, ao satisfazer as necessidades com relação à precisão, exatidão, linearidade e homogeneidade do intervalo de trabalho, foi validado, estando pronto para entrar em rotina no Laboratório Central da EPAL.

\section{REFERÊNCIAS}

1. von Guten, U.; Hoigné, J.; Environ. Sci. Technol. 1994, 28, 1234.

2. Ozekin, K.; Westerhoff, P.; Amy, G. L.; Siddiqui, M.; J. Environ. Eng. 1998, May, 456.
3. World Health Organization, Guidelines for Drinking Water Quality, $2^{\text {nd }}$ ed., vol. 2, Geneva, 1996; http://www.who.int/docstore/water_sanitation_health/ GDWQ/Chemicals/bromatefull.htm, acessada em Março 2003.

4. Environmental Protection Agency (U.S.EPA) Code of Federal Regulations Parts 9, 141, 142 National Primary Drinking Water Regulations: Disinfectants and Disinfection By-products; Final Rule 69406 Federal Register/vol. 63, no. 241/Wednesday, December 16, 1998; http:// www.epa.gov/OGWDW/mdbp/dbpfr.pdf, acessada em Março 2003.

5. Directiva 98/83/CE do Conselho, de 3 de Novembro de 1998, relativa à qualidade da água destinada ao consumo humano; Jornal Oficial das Comunidades Européias, L 330, 5 de Dezembro de 1998.

6. Hautman, D. P.; Munch, D. J.; Method 300.1, Determination of Inorganic Anions in Drinking Water by Ion Chromatography, Revision 1.0; National Exposure Research Laboratory, U.S. Environmental Protection Agency, Cincinnati, Ohio, 1997; http://www.epa.gov/safewater/methods/met300.pdf, acessada em Março 2003.

7. http://www.ktl.fi/sytty/abstracts/varti3.htm, acessada em Julho 2003.

8. ISO 8466-1 - Water Quality - Calibration and evaluation of analytical methods and estimation of performance characteristics - Part 1: Statistical evaluation of the linear calibration function, International Standard 1990.

9. ISO 8466-2 - Water Quality - Calibration and evaluation of analytical methods and estimation of performance characteristics - Part 2: Calibration strategy for non linear calibration functions, International Standard 1990.

10. Guia Relacre $n^{\circ} 13$ - Validação de Métodos Internos de Ensaio em Análise Química, Relacre, Lisboa, 2000 (ISBN 972-8574-02-9).

11. Standard Methods for the Examination of Water and Wastewater, $19^{\text {th }}$ ed. of Standard Methods, 1995. 